\title{
Fingerprint analysis of processed Rhizoma Chuanxiong by high-performance liquid chromatography coupled with diode array detection
}

\author{
Jia-Yan Fang ${ }^{1+}$, Lin Zhu ${ }^{1+}$, Tao Yi ${ }^{1 *}$, Jian-Ye Zhang ${ }^{2}$, Ling Yi ${ }^{1}$, Zhi-Tao Liang ${ }^{1}, \mathrm{Li} \mathrm{Xia}^{3}$, Jia-Fu Feng ${ }^{4}$, Jun Xu', \\ Yi-Na Tang ${ }^{1}$, Zhong-Zhen Zhao ${ }^{1}$ and Hu-Biao Chen $^{1 *}$
}

\begin{abstract}
Background: Rhizoma Chuanxiong (RC) is the dried rhizome of Ligusticum chuanxiong Hort., and various types of processed Rhizoma Chuanxiong (PRC) are widely used in China. However, quality assurance and quality control of these processed medicines remain challenging. This study aims to investigate the chemical compositions of various PRC preparations by a high-performance liquid chromatography (HPLC) coupled with diode array detection (DAD) method.
\end{abstract}

Methods: A HPLC-DAD method with validation was developed for PRC samples. Seven batches of plant samples from two processing methods, stir-frying and steaming, were analyzed by the HPLC-DAD method. Common peaks in PRC chromatograms were chosen to calculate their relative retention time (RRT) and relative peak area (RPA), and similarity analyses of the chromatographic fingerprints were conducted by Similarity Evaluation System for Chromatographic Fingerprint of Traditional Chinese Medicine software (Version 2004 A).

Results: In the 24-h stability test, the relative standard deviation for the RRT and RPA was less than $0.07 \%$ and $2.57 \%$, respectively. The precision was less than $0.08 \%$ for the RRT and $2.48 \%$ for the RPA. The repeatability for the RRT and RPA was less than $0.03 \%$ and $2.64 \%$, respectively. The similarities between the seven PRC batches were range from 0.956 to 0.990. After stir-frying or steaming, the amount of ferulic acid in PRC was much higher than that in the raw material.

Conclusions: The fingerprint analysis of PRC by different processing methods was feasible by HPLC-DAD.

Keywords: Rhizoma Chuanxiong, HPLC-DAD, Ligusticum chuanxiong, Fingerprint, Processing

\section{Background}

Many Chinese herbal medicines should be processed before clinical use, for achieving a unique function, reducing toxicity, enhancing efficacy, or stabilizing active ingredients $[1,2]$. Such processing involves many kinds of adjuvants. Rice wine and vinegar are the most widely used adjuvants [2]. Ginger juice, bran, and salt, are also often used [3,4]. Different processed products derived from the same botanic source are often used as the same herb.

\footnotetext{
* Correspondence: yitao@hkbu.edu.hk; hbchen@hkbu.edu.hk

${ }^{\dagger}$ Equal contributors

'School of Chinese Medicine, Hong Kong Baptist University, Hong Kong Special Administrative Region, Hong Kong, People's Republic of China
} Full list of author information is available at the end of the article
Hence, monitoring the quality of these processed medicines from different processing methods is critical [5].

Rhizoma Chuanxiong (RC), the dried rhizome of Ligusticum chuanxiong Hort. (Umbelliferae), is a major cardiovascular protective Chinese herb [6,7], especially for treating angina pectoris, cardiac arrhythmias, hypertension, and stroke $[8,9]$. RC also exhibits neuroprotective, anti-fibrotic, anti-nociceptive, anti-inflammatory, and antineoplastic activities [10-12]. Alkyl phthalides, phthalide dimers, and phenolic constituents were reported to be the main compounds responsible for the bioactivities and properties of RC [12-17]. Furthermore, some of the bioactive components were considered as drug candidates $[18,19]$. Ferulic acid, as a chemical marker of RC according

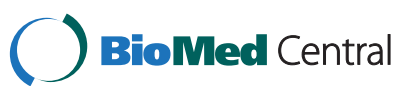

(c) 2015 Fang et al.; licensee BioMed Central. This is an Open Access article distributed under the terms of the Creative Commons Attribution License (http://creativecommons.org/licenses/by/4.0), which permits unrestricted use, distribution, and reproduction in any medium, provided the original work is properly credited. The Creative Commons Public Domain Dedication waiver (http://creativecommons.org/publicdomain/zero/1.0/) applies to the data made available in this article, unless otherwise stated. 
to the Chinese Pharmacopoeia, is a main bioactive constituent [20]. Through our recent market investigation on $\mathrm{RC}$, we found that stir-frying and steaming are the main processing methods, and that rice wine and vinegar are always used in the processing of RC.

Current analytical and quality control methods for RC simply focus on the detection of a few compounds by high-performance liquid chromatography (HPLC) and capillary electrophoresis [21,22]. Fingerprint analysis based on chromatography has been widely used for authentication and quality control of herbal drugs [23-26]. The combined HPLC and diode array detection (DAD) can provide online spectral information for each peak in a chromatogram, which has become a powerful tool for the rapid identification of the constituents in herbal products. Therefore, this study aims to investigate the chemical compositions of various processed RC (PRC) preparations by a HPLC coupled with DAD method.

\section{Methods \\ Materials}

Dried RC, which was produced in Sichuan, was purchased from Qingping Market of Guangzhou in mainland China. The authentication of the herbs was confirmed by Dr. Yi Tao according to the morphological features [6]. The dried rhizomes were sliced, and a total of seven batches of RC were obtained. Each batch had triplicate samples weighing $50 \mathrm{~g}$. The seven batches of the herb were processed under different processing conditions (Table 1) [27].

\section{Reagents and chemicals}

Rice wine was purchased from the Pagoda Brand (Zhejiang Pagoda Brand; Shaoxing Rice Wine Co. Ltd., China; alcohol: 15\%) and vinegar was purchased from the Amoy Brand (Amoy Food Ltd., Hong Kong; acetic acid: 5-8\%).

Ferulic acid was purchased from the National Institute for the Control of Pharmaceutical and Biological Products (China). The standard compounds of senkyunolide I, senkyunolide $\mathrm{H}$, senkyunolide $\mathrm{A}, \mathrm{Z}$-ligustilide, and levistolide A were isolated from $\mathrm{RC}$ in our laboratory [28], and their structures were shown in Figure 1.
The acetonitrile and formic acid used in the HPLC analysis were of HPLC grade and obtained from Lab-scan (Thailand). The methanol used for the sample extraction was of analysis grade and also purchased from Lab-scan (Thailand). Deionized water was generated using a MilliQ water purification system (Millipore, USA).

\section{HPLC- DAD instrumentation and conditions}

HPLC-DAD was carried out by an Agilent 1100 series HPLC-DAD system comprising a vacuum degasser, binary pump, autosampler, thermostated column compartment, and DAD (Agilent, USA), which was used for acquiring chromatograms and UV spectra. An Alltima $\mathrm{C}_{18}$ column $(5 \mu \mathrm{m} ; 4.6 \times 250 \mathrm{~mm})$ was used for HPLC analysis. The mobile phase consisted of $0.1 \%$ formic acid in water (A) and acetonitrile (B), and the procedure was performed with a gradient program of $15-20 \%$ (B) at $0-10 \mathrm{~min}, 20-$ $53 \%$ (B) at $10-40 \mathrm{~min}$, and $53-100 \%$ (B) at $40-60 \mathrm{~min}$. The flow rate was $1 \mathrm{~mL} / \mathrm{min}$. The detection wavelength was set at $280 \mathrm{~nm}$. The column temperature was set at $30^{\circ} \mathrm{C}$. The injection volume was $10.0 \mu \mathrm{L}$.

\section{Preparation of standard and sample solutions}

Standard solutions were prepared at a concentration of $50 \mathrm{mg} / \mathrm{L}$ with methanol. The seven batches of PRC were crushed with a grinder. The ground powder was passed through a 20 -mesh $(0.85-\mathrm{mm})$ sieve and stored at about $4^{\circ} \mathrm{C}$ before use. PRC powder $(0.25 \mathrm{~g})$ was immersed in $8 \mathrm{~mL}$ of methanol and ultrasonically extracted for $30 \mathrm{~min}$ at room temperature, and this procedure was repeated three times. The mixture was centrifuged (Eppendorf $5810 \mathrm{R}$, Hamburg, Germany) at $3200 \times g$ for $10 \mathrm{~min}$. The supernatant solution was transferred into a 25 -mL volumetric flask, made up to full volume with methanol, and filtered through a nylon syringe filter $(0.20 \mu \mathrm{m}$, Filtrex, USA) before HPLC analysis.

\section{Assay validation and sample determination}

The method precision was determined by injecting the same extract of the PRC-0 sample six times in one day. The method repeatability was evaluated by analyzing six independently-extracted PRC-0 samples. The sample

Table 1 Seven processing methods for PRC $(n=3)$

\begin{tabular}{llll}
\hline Samples & Processing methods & Additive and ratio (w:w) & Processing time (h) \\
\hline PRC-0 & - & - & - \\
PRC-1 & Stir-frying & - & 0.5 \\
PRC-2 & Steaming & - & 0.5 \\
PRC-3 & Stir-frying with rice wine & Rice wine (1:10) & 0.5 \\
PRC-4 & Steaming with rice wine & Rice wine (1:10) & 0.5 \\
PRC-5 & Stir-frying with vinegar & Vinegar (1:5) & 0.5 \\
PRC-6 & Steaming with vinegar & Vinegar (1:5) & 0.5 \\
\hline
\end{tabular}

*The ( $w: w)$ values are the weight ratios of the additive to the herb. 
<smiles>COc1cc(/C=C/C(=O)O)ccc1O</smiles>

Ferulic acid (2)<smiles>CCC/C=C1\OC(=O)C2=C1CCC(O)C2O</smiles>

Senkyunolide I (4)<smiles>C/C=C1\OC(=O)C2=C1CCC(O)C2O</smiles>

Senkyunolide H (5)<smiles>CCC(O)C=C1OC(=O)C2CCCCC12</smiles>

Senkyunolide F (6)<smiles>CCCCC1OC(=O)C2=C1CCC=C2</smiles>

Senkyunolide A (8)<smiles>CCC/C=C1\OC(=O)C2=C1CCC=C2</smiles>

Z-Ligustilide (11)

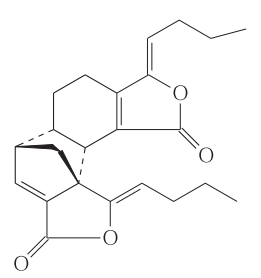

Levistolide A (15)

Z-Butylidenephthalide (12)

Riligustilide (13)

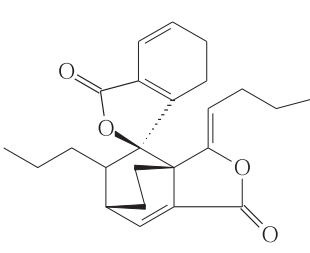

Tokinolide B (14)

Figure 1 Structures of the identified compounds in the fingerprints of the PRC samples.

stability was assessed by the same PRC-0 extract at 0,2 , $4,8,16$, and $24 \mathrm{~h}$. The similarity evaluation of PRC was performed on seven batches of processed samples.

The data analysis was conducted by Similarity Evaluation System for Chromatographic Fingerprint of Traditional Chinese Medicine software (Version 2004 A), which was recommended by the State Food and Drug Administration of China. The software was used to calculate the correlation coefficients of the chromatographic profiles of the seven batches of PRC samples, and to generate a simulative mean chromatogram (SMC). The similarities of different chromatographic fingerprints were compared by the PRC0 samples.

\section{Results and discussion}

Identification of major constituents in various PRC samples Typical chromatogram of the PRC was shown in Figure 2. Based on comparisons with standard compounds, six peaks were identified as feruled acid (2), senkyunolide I (4), senkyunolide H (5), senkyunolide A (8), Z-ligustilide

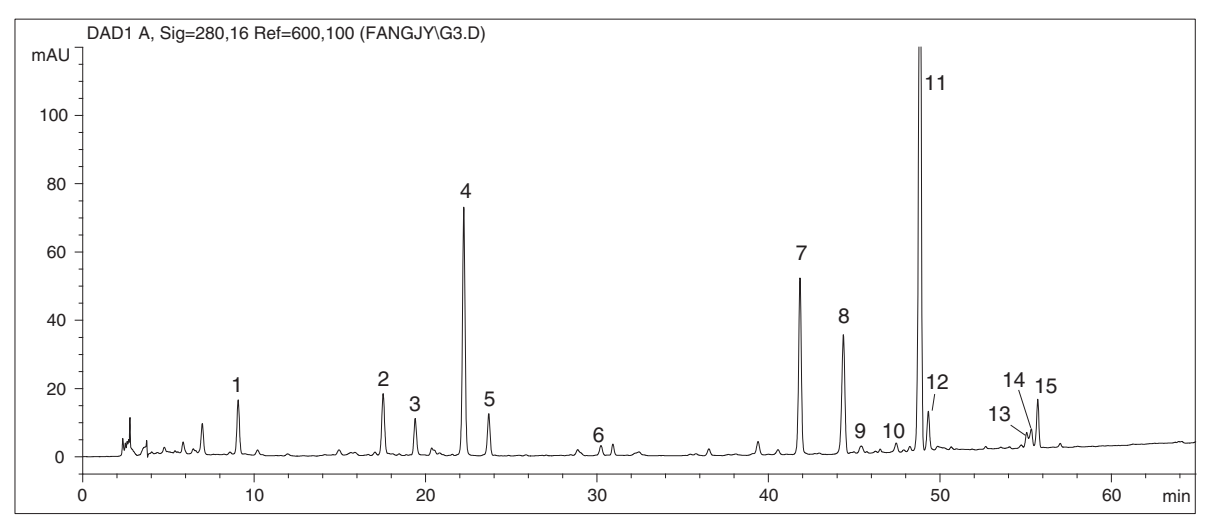

Figure 2 Typical chromatogram of the PRC-0 sample at $280 \mathrm{~nm}$. 
Table 2 Validation of the fingerprint method $(n=3)$

\begin{tabular}{|c|c|c|c|c|c|c|}
\hline \multirow[b]{2}{*}{ Peak No. } & \multicolumn{2}{|c|}{ Stability (RSD, \%) } & \multicolumn{2}{|c|}{ Precision (RSD, \%) } & \multicolumn{2}{|c|}{ Repeatability (RSD, \%) } \\
\hline & RRT & RPA & RRT & RPA & RRT & RPA \\
\hline 1 & 0.04 & 2.06 & 0.06 & 2.07 & 0.03 & 0.67 \\
\hline 2 & 0.06 & 2.57 & 0.07 & 2.40 & 0.02 & 1.14 \\
\hline 3 & 0.07 & 2.57 & 0.08 & 2.25 & 0.02 & 1.55 \\
\hline 4 & 0.05 & 1.98 & 0.06 & 1.79 & 0.03 & 0.66 \\
\hline 5 & 0.05 & 1.96 & 0.05 & 1.77 & 0.03 & 0.83 \\
\hline 6 & 0.03 & 1.81 & 0.04 & 2.05 & 0.01 & 1.09 \\
\hline $7(S)$ & - & - & - & - & - & - \\
\hline 8 & 0.01 & 1.89 & 0.00 & 1.69 & 0.01 & 0.69 \\
\hline 9 & 0.01 & 1.80 & 0.00 & 2.48 & 0.01 & 2.32 \\
\hline 10 & 0.01 & 2.45 & 0.01 & 2.31 & 0.01 & 2.20 \\
\hline 11 & 0.01 & 1.93 & 0.01 & 1.73 & 0.02 & 0.46 \\
\hline 12 & 0.01 & 2.15 & 0.01 & 2.44 & 0.02 & 2.19 \\
\hline 13 & 0.02 & 1.33 & 0.01 & 0.65 & 0.03 & 1.77 \\
\hline 14 & 0.02 & 1.26 & 0.01 & 1.32 & 0.03 & 2.64 \\
\hline 15 & 0.02 & 1.60 & 0.02 & 2.04 & 0.03 & 1.72 \\
\hline
\end{tabular}

(11), and levistolide A (15). Seven other peaks were tentatively identified as senkyunolide F (6), coniferyl ferulate (7), butylphthalide (9), E-ligustilide (10), Z-butylidenephthalide (12), riligustilide (13), and tokinolide B (14) by comparing their retention times and UV spectra with the standard compounds. From the assay results, the major constituents in the various PRC samples were similar, namely phenolic constituents, alkylphthalides, and phthalide dimers.
Validation of the fingerprint analysis method

The fingerprints of the PRC samples were obtained by HPLC in 60 min. Fifteen common peaks were found in the HPLC fingerprint chromatograms of the seven batches of PRC samples. Peak 7 (coniferyl ferulate) was assigned as the reference peak to conduct method validation, because it had a moderate retention time and peak area compared with the other peaks. The HPLC method was validated in terms of stability, precision, and

Table 3 RRT and RPA of common peaks in seven batches of PRC $(n=3)$

\begin{tabular}{|c|c|c|c|c|c|c|c|c|c|c|c|c|c|c|c|c|c|c|}
\hline \multirow[t]{2}{*}{ Peak No. } & \multicolumn{2}{|c|}{ PRC-0 } & \multicolumn{2}{|c|}{ PRC-1 } & \multicolumn{2}{|c|}{ PRC-2 } & \multicolumn{2}{|c|}{ PRC-3 } & \multicolumn{2}{|c|}{ PRC-4 } & \multicolumn{2}{|c|}{ PRC-5 } & \multicolumn{2}{|c|}{ PRC-6 } & \multicolumn{2}{|c|}{ Average } & \multicolumn{2}{|c|}{ RSD (\%) } \\
\hline & RRT & $\overline{\mathrm{RPA}}$ & RRT & $\overline{\mathrm{RPA}}$ & RRT & $\overline{\mathrm{RPA}}$ & RRT & $\overline{\mathrm{RPA}}$ & RRT & $\overline{\mathrm{RPA}}$ & RRT & $\overline{\mathrm{RPA}}$ & RRT & $\overline{\mathrm{RPA}}$ & RRT & $\overline{\mathrm{RPA}}$ & RRT & $\overline{R P A}$ \\
\hline 1 & 0.22 & 0.20 & 0.21 & 0.27 & 0.21 & 0.69 & 0.21 & 0.30 & 0.22 & 1.46 & 0.21 & 0.46 & 0.21 & 0.63 & 0.21 & 0.57 & 0.39 & 75.7 \\
\hline 2 & 0.42 & 0.28 & 0.42 & 0.68 & 0.42 & 1.30 & 0.42 & 0.55 & 0.42 & 2.97 & 0.42 & 1.48 & 0.42 & 1.11 & 0.42 & 1.20 & 0.05 & 74.6 \\
\hline 3 & 0.46 & 0.16 & 0.46 & 0.23 & 0.46 & 0.34 & 0.46 & 0.20 & 0.46 & 0.68 & 0.46 & 0.57 & 0.46 & 0.42 & 0.46 & 0.37 & 0.76 & 53.2 \\
\hline 4 & 0.53 & 1.07 & 0.53 & 2.00 & 0.53 & 3.10 & 0.53 & 1.83 & 0.53 & 6.64 & 0.53 & 3.78 & 0.53 & 2.73 & 0.53 & 3.02 & 0.04 & 60.4 \\
\hline 5 & 0.57 & 0.18 & 0.57 & 0.34 & 0.57 & 0.52 & 0.57 & 0.30 & 0.57 & 1.13 & 0.57 & 0.62 & 0.57 & 0.47 & 0.57 & 0.51 & 0.03 & 61.1 \\
\hline 6 & 0.72 & 0.05 & 0.72 & 0.08 & 0.72 & 0.12 & 0.72 & 0.07 & 0.72 & 0.25 & 0.72 & 0.14 & 0.72 & 0.11 & 0.72 & 0.12 & 0.02 & 55.2 \\
\hline $7(S)$ & 1 & 1 & 1 & 1 & 1 & 1 & 1 & 1 & 1 & 1 & 1 & 1 & 1 & 1 & 1 & 1 & - & - \\
\hline 8 & 1.06 & 0.70 & 1.06 & 1.14 & 1.06 & 1.95 & 1.06 & 1.01 & 1.06 & 3.31 & 1.06 & 1.78 & 1.06 & 1.68 & 1.06 & 1.65 & 0.01 & 52.1 \\
\hline 9 & 1.09 & 0.05 & 1.09 & 0.07 & 1.09 & 0.11 & 1.09 & 0.06 & 1.09 & 0.23 & 1.09 & 0.13 & 1.09 & 0.10 & 1.09 & 0.11 & 0.03 & 57.6 \\
\hline 10 & 1.13 & 0.06 & 1.13 & 0.09 & 1.13 & 0.14 & 1.13 & 0.07 & 1.13 & 0.26 & 1.13 & 0.16 & 1.13 & 0.12 & 1.13 & 0.13 & 0.02 & 53.4 \\
\hline 11 & 1.17 & 3.01 & 1.17 & 3.73 & 1.17 & 6.81 & 1.17 & 3.76 & 1.17 & 13.9 & 1.17 & 6.50 & 1.17 & 6.23 & 1.17 & 6.28 & 0.02 & 58.9 \\
\hline 12 & 1.18 & 0.12 & 1.18 & 0.16 & 1.18 & 0.25 & 1.18 & 0.15 & 1.18 & 0.57 & 1.18 & 0.29 & 1.18 & 0.23 & 1.18 & 0.25 & 0.02 & 60.0 \\
\hline 13 & 1.31 & 0.02 & 1.32 & 0.12 & 1.32 & 0.17 & 1.32 & 0.10 & 1.32 & 0.36 & 1.32 & 0.24 & 1.32 & 0.16 & 1.32 & 0.17 & 0.22 & 65.1 \\
\hline 14 & 1.32 & 0.08 & 1.32 & 0.13 & 1.32 & 0.23 & 1.32 & 0.12 & 1.32 & 0.47 & 1.32 & 0.27 & 1.32 & 0.20 & 1.32 & 0.21 & 0.19 & 61.0 \\
\hline 15 & 1.33 & 0.23 & 1.33 & 0.33 & 1.33 & 0.51 & 1.33 & 0.29 & 1.33 & 1.03 & 1.33 & 0.65 & 1.33 & 0.44 & 1.33 & 0.50 & 0.04 & 55.4 \\
\hline
\end{tabular}




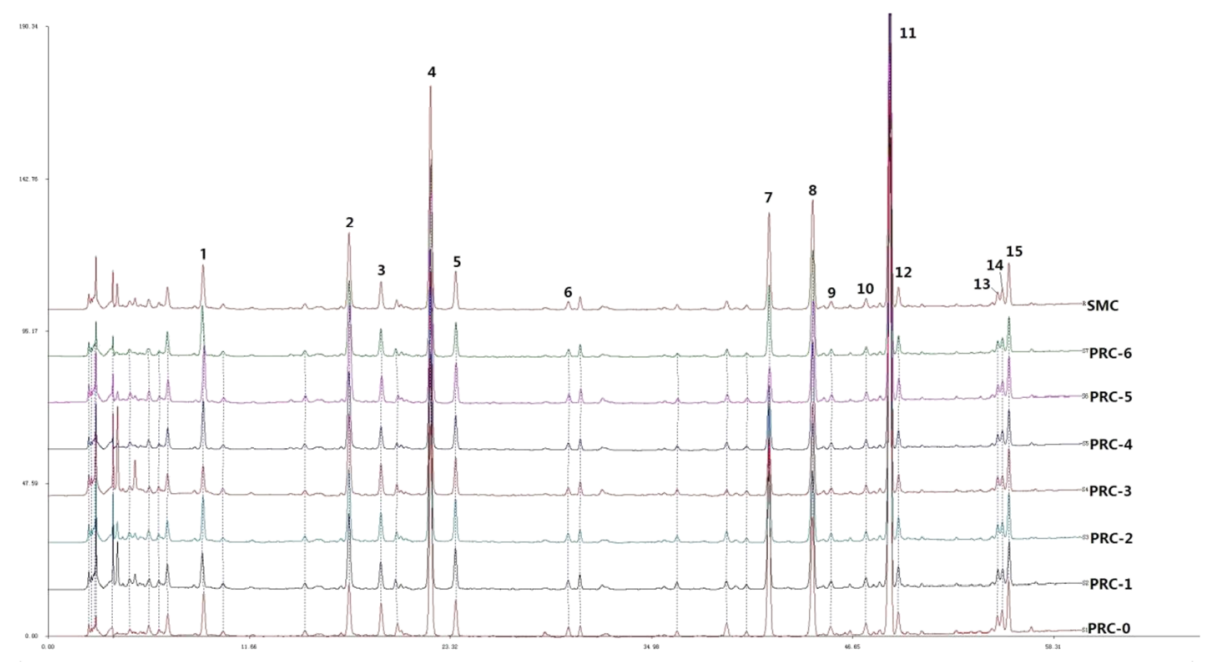

Figure 3 HPLC-DAD fingerprints of seven batches of PRC and SMC at $280 \mathrm{~nm}$.

repeatability, and the results were listed in Table 2 . In the 24-h stability test, the relative standard deviation (RSD) of the relative retention time (RRT), defined as the ratio of the retention time of the individual peak to that of the reference peak, and the relative peak area (RPA), defined as the ratio of the retention peak area of the individual peak to that of the reference peak, was less than $0.07 \%$ and $2.57 \%$, respectively. The precision was less than $0.08 \%$ for the RRT and $2.48 \%$ for the RPA. The repeatability for the RRT and RPA was less than $0.03 \%$ and $2.64 \%$, respectively. These data indicated that the HPLC method was suitable for fingerprint analysis of various PRC samples.

\section{Fingerprint analysis of various PRC samples}

Seven batches of PRC samples were analyzed with the present method. Using the Similarity Evaluation System for Chromatographic Fingerprint of Traditional Chinese Medicine (Version 2004 A), the RRT and RPA of the 15 common fingerprint peaks were calculated, and the results are shown in Table 3 with their mean and RSD values, respectively. The RSD values for the RRT fell in the range of $0.01-0.76 \%$, while the RSD values of the RPA were observed in the range of $52.1-75.7 \%$. The RSD values for the RRT were less than $0.76 \%$, indicating that the fingerprint analysis was precise. The RSD values for the RPA were much larger, demonstrating that the processing methods had affected the contents of the compounds in the PRC samples. Although the contents varied, this fingerprint analysis by HPLC was still feasible and repeatable.

The chromatograms of the PRC samples from the seven processing methods and the SMC were shown in Figure 3. The results of the similarity analysis were listed in Table 4. The similarities between the seven batches of
PRC samples were found to range from 0.956 to 0.990 (with PRC-0 serving as a reference), and the influences of processing on $\mathrm{RC}$ were as follows: PRC-5 (stir-frying with vinegar) $>\mathrm{PRC}-4$ (steaming with wine) $>\mathrm{PRC}-2$ (steaming) $>$ PRC-1 (stir-frying) $>$ PRC-6 (steaming with vinegar) $>$ PRC-3 (stir-frying with wine) $>$ PRC-0 (raw material). From these results, the processing methods of stir-frying with vinegar and steaming with wine caused significant differences in the chemical composition of $\mathrm{RC}$ after processing. From Table 3, the RPA values of the 15 constituents (with peak 7 assigned as a reference) in the various PRC samples all increased, compared with those in the raw material, indicating that the amounts of the 15 compounds in $\mathrm{RC}$ increased after processing.

As shown in Figure 3, the chromatogram revealed that peak 7 (coniferyl ferulate) was one of the major compounds in raw RC. Coniferyl ferulate was found to be susceptible to hydrolysis into ferulic acid in neutral, strongly acidic, and basic solvents, where heat and water could facilitate the hydrolysis [29]. This could result in the variable amounts of ferulic acid determined. In the present study, the ratios of ferulic acid to coniferyl ferulate in $\mathrm{RC}$ after processing with different methods were

Table 4 Similarities of seven batches of PRC

\begin{tabular}{ll}
\hline Batch & Similarity \\
\hline PRC-0 & 1.000 \\
PRC-1 & 0.980 \\
PRC-2 & 0.978 \\
PRC-3 & 0.990 \\
PRC-4 & 0.963 \\
PRC-5 & 0.956 \\
PRC-6 & 0.982 \\
\hline
\end{tabular}


much higher than that in the raw material. This could also be explained by the increase in the amount of ferulic acid in $\mathrm{RC}$ after processing. Ferulic acid is known to be the active component of PRC and responsible for its therapeutic effects, and the enhancement of ferulic acid could increase its biological effects in herbs $[1,6]$.

Many Chinese herbal medicines need to be processed before clinic use. However, the study on the quality assurance and quality control (QA \& QC) of these processed medicines is still inadequate. In our manuscript, a fingerprint analysis by HPLC-DAD method was developed and well validated to evaluate PRC, a typical Chinese herbal medicine. Base on the present study, our result indicates HPLC-DAD fingerprinting is a powerful approach for QA \& QC of PRC, which can be applied to other processed medicines. Thus, this study contributes to the quality study of Chinese medicines, especially for the research of processed Chinese medicine by HPLC-DAD fingerprinting.

\section{Conclusions}

The fingerprint analysis of PRC by different processing methods were feasible by HPLC-DAD.

\section{Abbreviations}

HPLC: High performance liquid chromatography; DAD: Diode array detection; PRC: Processed Rhizoma Chuanxiong; RRT: Relative retention time; RPA: Relative peak area; RSD: Relative standard deviation; QA: Quality assurance; QC: Quality control.

\section{Competing interests}

The authors declare that they have no competing interests.

\section{Authors' contributions}

All authors designed the study. JYF, LZ, JYZ, LY and ZTL performed the experiments. JFF, LX, JX, YNT and ZZZ analyzed the data and drafted the data reports. JYF and LZ wrote the manuscript. All authors read and approved the final version of the manuscript.

\section{Author details \\ 'School of Chinese Medicine, Hong Kong Baptist University, Hong Kong Special Administrative Region, Hong Kong, People's Republic of China. ${ }^{2}$ School of Pharmaceutical Sciences, Guangzhou Medical University, Guangzhou 510182, People's Republic of China. ${ }^{3}$ School of Traditional Chinese Medicine, Guangdong Food and Drug Vocational College, Guangzhou 510520, People's Republic of China. ${ }^{4}$ Leshan Pharmaceutical Research Center, Leshan Vocational \& Technical College, Leshan 614000, People's Republic of China.}

Received: 4 August 2014 Accepted: 26 January 2015

Published online: 10 February 2015

\section{References}

1. Zhan JY, Zheng KY, Zhu KY, Bi CW, Zhang WL, Du CY, et al. Chemical and biological assessment of Angelicae Sinensis Radix after processing with wine: an orthogonal array design to reveal the optimized conditions. J Agric Food Chem. 2011;59:6091-8.

2. Wang $Q$, Wu YK. Recent situation of Chinese medicine processing. J Liaoning Univ TCM. 2012;14:227-8.

3. Xie ZD, Yi DY, Fang YQ, Guo JS. Studies on traditional pharmaceutical processing for chuanxiong rhizome in medical history and modern research. Chin J Exp Tradit Med Formulae. 2012;18:290-3.
4. Xia Q, Wen HL, Li TG, He JE. Determination of free ferulic acid and total ferulic acid in different processed products of Rhizoma Chuanxiong by HPLC. J Guangzhou Univ Tradit Chin Med. 2009;26:384-7.

5. Zhao ZZ, Liang ZT, Kelvin C, Lu GH, Lee LM, Chen HB, et al. A unique issue in the standardization of Chinese materia medica: processing. Plant Med. 2010;76:1975-86.

6. The State Pharmacopoeia Commission of P. R. China. Pharmacopoeia of the People's Republic of China Vol. 1. Beijing: Chemical Industry Press; 2010. p. 38.

7. Hu SL. The authentic and superior medicinal herbals in China. Harbin, China: Heilongjiang Science and Technology Publishing House; 1989. p. 461-3.

8. Wang $Y$ H, Liang S, Xu DS, Lin X, He CY, Feng Y, et al. Effect and mechanism of senkyunolide I as an anti-migraine compound from Ligusticum chuanxiong. J Pharma Pharmacol. 2011;63:261-6.

9. Li SL, Chan SSK, Lin G, Ling L, Yan R, Chung HS, et al. Simultaneous analysis of seventeen chemical ingredients of Ligusticum chuanxiong by on-line high performance liquid chromatography-diode array detector-mass spectrometry. Planta Med. 2003;69:445-51.

10. Ran X, Ma L, Peng C, Zhang H, Qin LP. Ligusticum chuanxiong Hort: a review of chemistry and pharmacology. Pharm Biol. 2011;49:1180-9.

11. Jin YQ, Hong YL, Li JR, Li X, Wang XX, Lu GH. Advancements in the chemical constituents and pharmacological effects of chuanxiong. Pharma Clin Chin Materia Medica. 2013;4:44-8.

12. Xu X, Ye T, Shuo Y, Yao L, Guang T. Anti-cancer effects of Ligusticum chuanxiong Hort alcohol extracts on HS766T cell. Afr J Tradit Complement Alter Med. 2013;10:542-6.

13. Erqun $\mathrm{S}, \mathrm{Xu} \mathrm{L}$. Rapid fingerprint analysis of Ligusticum chuanxiong by UFLC-DAD. J Chromatogr Sci. 2013;51:331-4

14. Yi T, Kelvin Leung SY, Lu GH, Zhang H, Kelvin C. Identification and comparative determination of sekyunolide $\mathrm{A}$ in traditional chinese medicine plants Ligusticum chuanxiong and Angelica sinensis by HPLC with DAD and ESI-MS. Chem Pharm Bull. 2005;53:1480-3.

15. Li SL, Yan R, Tam YK, Lin G. Post-harvest alteration of the main chemical ingredients in Ligusticum chuanxiong Hort.(Rhizoma Chuanxiong). Chem Pharm Bull. 2007:55:140-4.

16. Li SL, Lin G, Tam YK. Time-course accumulation of main bioactive components in the rhizome of Ligusticum chuanxiong. Planta Med. 2006;72:278-80.

17. Li W, Tang Y, Chen Y, Duan JA. Advances in the chemical analysis and biological activities of chuanxiong. Molecules. 2012;17:10614-51.

18. Or CT, Yang LH, Law HY, Li CB, Lau SY. Isolation and identication of anti-inflammatory constituents from Ligusticum chuanxiong and their underlying mechanisms of action on microglia. Neuropharmacology. 2011;60:823-31.

19. Huang J, Lu XQ, Lu J, Li GY, Wang HY, Li LH, et al. Two new phthalides with BuChE inhibitory activity from Ligusticum chuanxiong. J Asian Nat Prod Res. 2013;15:1237-42.

20. Chen $X Q$, Kong L, Su XY, Fu HJ, Ni JY, Zhao RH, et al. Separation and identification of compounds in Rhizoma chuanxiong by comprehensive two-dimensional liquid chromatography coupled to mass spectrometry. J Chromatogr A. 2004;1040:169-78.

21. Li HX, Ding MY, LV K, Yu JY. Determination of the active ingredients in Chuanxiong by HPLC, HPLC-MS, and El-MS. J Liquid Chromatog Related Technol. 2001;24:2017-31.

22. Ji SG, Chai YF, Wu YT, Yin XP, Liang DS, Xu ZM, et al. Determination of ferulic acid in Angelica sinensis and Chuanxiong by capillary zone electrophoresis. Biomed Chromatogr. 1999;13:333-4.

23. Yi T, Chen QL, He XC, So SW, Lo YL, Fan LL, et al. Chemical quantification and antioxidant assay of four active components in Ficus hirta root using UPLC-PAD-MS fingerprinting combined with cluster analysis. Chem Cent J. 2013;7:115.

24. Qiao CF, Han QB, Song JZ, Mo SF, Kong LD, Kung HF, et al. Chemical fingerprint and quantitative analysis of fructus psoraleae by high-performance liquid chromatography. J Sep Sci. 2007;30:813-8.

25. Qin XM, Dai YT, Zhang LZ, Guo XQ, Shao HX. Discrimination of three medicinal materials from the Citrus genus by HPLC fingerprint coupled with two complementary software. Phytochem Anal. 2009;20:307-13.

26. Yi T, Chen HB, Zhao ZZ, Yu ZL, Jiang ZH. Comparison of the chemical profiles and anti-platelet aggregation effects of two "Dragon's Blood" drugs used in traditional Chinese medicine. J Ethnopharmacol. 2011;133:796-802.

27. People's Republic of China Ministry of Health, Bureau of Pharmaceutical Affairs. Processing standards of the People's Republic of China. Beijing: People's Health Press; 1988. p. 14. 
28. Yi T, Leung KSY, Lu GH, Chan KC, Zhang H. Simultaneous qualitative and quantitative analyses of the major constituents in the rhizome of Ligusticum chuanxiong using HPLC-DAD-MS. Chem Pharm Bull. 2006;54:255-9.

29. Lu GH, Chan K, Leung K, Chan CL, Zhao ZZ, Jiang ZH. Assay of free ferulic acid and total ferulic acid for quality assessment of Angelica sinensis. J Chromatogr A. 2005;1068:209-19.

Submit your next manuscript to BioMed Central and take full advantage of:

- Convenient online submission

- Thorough peer review

- No space constraints or color figure charges

- Immediate publication on acceptance

- Inclusion in PubMed, CAS, Scopus and Google Scholar

- Research which is freely available for redistribution 